\title{
Topography of the Retina and Striate Cortex and its Relationship to Visual Acuity in Rhesus Monkeys and Squirrel Monkeys*
}

\author{
E.T. Rolls and A. CoweY \\ Institute of Experimental Psychology, University of Oxford (England)
}

Received October 6, 1969

Summary. Density of cones and ganglion cells was studied in horizontal sections of retina in the rhesus monkey (Macaca mulatta) and the squirrel monkey (Saimiri sciureus). The lower angular density of cones in the fovea of Saimiri correlates with its visual acuity, which is poorer than that of Macaca $(0.74$ and $0.65 \mathrm{~min}$ of arc respectively). Cone density falls more steeply with angular eccentricity from the fovea in Saimiri, in accordance with its relatively poorer peripheral acuity. Comparable results were obtained with retinal ganglion cells, but the comparisons at the fovea itself are more difficult because of the lateral displacement of these elements in the foveal region.

The cortical magnification of the visual field (that is, the number of $\mathrm{mm}$ of cortex per degree of visual field) is lower for both the foveal and parafoveal representations at the striate cortex in Saimiri. This was correlated with its poorer foveal and parafoveal acuity. It was shown that with increasing eccentricity from the fovea, the fall in the magnification of the visual field at the striate cortex is approximately proportional to the decrease in ganglion cell density at the retina. The results of this study, in which acuity and topography of the visual system are compared in two species of monkey, are consistent with the view that both retinal topography, and the cortical magnification of the visual field, are closely related to visual acuity.

Key Words : Visual acuity — Cones - Ganglion cells — Striate cortex

\section{Introduction}

It is well established that in man visual acuity falls monotonically with increasing eccentricity of the test stimulus from the fovea. There may be an explanation for this relationship in terms of the anatomy of the visual system, for with increasing eccentricity from the fovea Østerberg (1935) has noted a fall in cone density, Weymouth (1958) and van Buren (1963) have found a monotonic decrease in ganglion cell density, and Daniel and Whitteridge (1961) have found that the magnification factor at the visual striate cortex in monkeys falls monotonically. The magnification factor (M) is the number of $\mathrm{mm}$ of cortex measured linearly along the cortex representing each linear degree of visual field.

* This work was supported by Medical Research Council Grant G.967/2/B. We wish to thank Mrs L. Bowman and Mr D. Canwell for their help in preparing histological material. 
If these anatomical and electrophysiological expressions really provide a basis for the variation of visual acuity across the visual field certain predictions should be fulfilled. One is that two species with similar foveal acuity should have equally similar cortical magnification factors for the fovea. This has been demonstrated for rhesus monkeys (Macaca mulatta) and squirrel monkeys (Saimiri sciureus) where foveal acuity is 0.65 and $0.74 \mathrm{~min}$ of arc respectively (Cowey and Ellis, 1967) and the central one degree of retina is represented by $6-8 \mathrm{~mm}$ of striate cortex (Whitteridge, personal communication) and $5-6 \mathrm{~mm}$ (Cowey, 1964) respectively. These two species would also be expected to possess similar cone and ganglion cell densities per unit angle associated with the fovea.

The cortical magnification factors for the extrafoveal retina in Macaca and Saimiri are very dissimilar, the extrafoveal retina being less well represented in the latter (Cowey, 1964). For example, although the fovea is similarly represented in the two species and cell density and depth of the striate cortex are the same in both, the total extent of striate cortex of one hemisphere is $1380 \mathrm{sq} . \mathrm{mm}$ in Macaca but only $720 \mathrm{sq}$.mm in Saimiri. As expected, peripheral acuity is poorer in Saimiri (Cowey and Ellis, 1969). It also follows that the cone and ganglion cell density in the extrafoveal retina should be greater in Macaca than in Saimiri.

In this paper the predictions about foveal and extrafoveal cone and retinal ganglion cell densities are examined. The acuity differences between the two species are also related to the cortical magnification factor of the visual field at different eccentricities in the two species. The variation of $M$ with eccentricity in a group of monkeys including rhesus and baboons has been investigated by Daniel and Whitteridge (1961) and is presented here for Saimiri.

\section{Materials and Methods}

\section{Retinal Ganglion Cell and Cone Densities in Saimiri and Macaca}

Five eyes of three juvenile male rhesus monkeys (weight $6 \mathrm{~kg}$ ) and five eyes of three juvenile male squirrel monkeys (weight $0.8 \mathrm{~kg}$ ) were perfused with formol-saline followed by Susa fixative, immediately after giving the animal a lethal intravenous dose of pentobarbitone. After enucleation they were left in the fixative for at least one week. Fluid exchange during the final stage of dehydration was facilitated either by removing the anterior portions of the eyes, or by making a cut through the sclera into the vitreous humour. The eyes were embedded in parafin, using the optic nerve stump and the muscle attachments to orientate the eyes so that $6 \mu$ serial sections could be cut which were in the plane of the horizontal retinal meridian. Sections were stained by a modified Masson method, using Haemalum-Ponceau S-Light Green.

Four other eyes had to be rejected either because the receptor elements were not cut in the appropriate plane or because the retina was deformed by the histological procedures.

Sections were examined under oil immersion at a magnification of 1000 diameters. Using an eye-piece graticule calibrated against a stage micrometer, cones and ganglion cell nuclei were counted in strips of $100 \mu$ starting from the centre of the fovea, which was taken as zero eccentricity. Counts thus represent the number of cones or ganglion cell nuclei in a strip of retina $100 \mu$ long $\times 6 \mu$ deep. Because care had been taken in the orientation of the eyes, the sections passed through the long axes of the cones, thus preventing the apparent multiplication of elements which occurs with oblique sections.

The counts were transformed into angular terms so that a comparison of the eyes could be made which was not affected by their different diameters, and in order that a comparison could be made with acuity thresholds. Firstly, the length of retina, in $\mathrm{mm}$, corresponding to one degree in the visual field was calculated from the positions of the nodal points. The latter were determined by scaling down in proportion to eye diameter, the position of the nodal point given by Duke-Elder (1932, 1, p. 740) for the human eye. The human eye diameter was taken 
as $24.13 \mathrm{~mm}$ and the posterior nodal distance as $17.05 \mathrm{~mm}$. Thus the posterior nodal distance (p.n.d.) for the rhesus monkeys, with a diameter of $20.0 \mathrm{~mm}$ (Cowey and Ellis, 1967) is 14.13 $\mathrm{mm}$, and the value for the squirrel monkey with an eye diameter of $14.5 \mathrm{~mm}$ (Cowey and Ellis, 1967 ) is $10.24 \mathrm{~mm}^{*}$. These values were checked by calculating the positions of the optical centres of aphakic monkey eyes as follows. The radius of curvature of two rhesus monkey corneae was measured from corneal moulds and found to be $6.5 \mathrm{~mm}$. Thus the equivalent of the p.n.d. for the aphakic optical system is $20.0-6.5$, or $13.5 \mathrm{~mm}$. The "aphakic p.n.d." is therefore $0.63 \mathrm{~mm}$ posterior to the calculated position of the p.n.d. of the optical system including the lens. For the squirrel monkey, the radius of curvature of the cornea was found to be $4.75 \mathrm{~mm}$, giving an "aphakic p.n.d." of $9.75 \mathrm{~mm}$. For this species the difference between the p.n.d's is thus 0.49 $\mathrm{mm}$. Using a value for the radius of curvature of the human cornea calculated according to Duke-Elder (1932, $I$, pp. 682-742), the difference between the two human p.n.d's was found to be $0.87 \mathrm{~mm}$. For each species this difference between the two p.n.d's follows eye diameter very closely, and shows that the p.n.d's calculated for the monkey eyes are reasonable. Using these p.n.d's, the length of the retina corresponding to one degree in the visual field is $0.246 \mathrm{~mm}$ for the rhesus monkey, and $0.180 \mathrm{~mm}$ for the squirrel monkey.

Next it was necessary to calculate the shrinkage which had occurred during the histological preparation of the eyes. Cowey (1967), using a perimeter and behavioural plotting of the blind spot, and Weiskrantz and Cowey (1967), using a measuring ophthalmoscope, found that in rhesus monkeys the angular separation of fovea and closest edge of optic disc was $15^{\circ}$. This distance in the sectioned eye was measured under the microscope, and the shrinkage was calculated from the length of retina corresponding to the angle of $15^{\circ}$. Shrinkage was also calculated from the changes in circumference of the eyes which occurred during preparation. The values found agreed closely in giving a value of $23 \%$ for linear shrinkage. This compares excellently with Østerberg's (1935) calculation of $24 \%$ linear shrinkage for human eyes prepared in a comparable way.

Using the values of $\mathrm{mm} /{ }^{\circ}$ and shrinkage as determined above, the angular measure calculated was the square root of the reciprocal of the number of cones or ganglion cells in a retinal area of 1 solid minute. This is a measure of separation, with dimensions minutes per cone or ganglion cell. For Macaca, separation $=7.76 / \sqrt{\mathrm{x}}$ where $\mathrm{x}=$ the count of cone or ganglion cells in 600 sq. $\mu$; for Saimiri, separation $=10.6 / \sqrt{\bar{x}}$.

A correction for the apparent multiplication of nuclei counted in thin sections was applied by the method of Abercrombie (Abercrombie, 1946) to the counts of ganglion cell nuclei. The correction applied throughout was for $5 \mu$ nuclei counted in $6 \mu$ sections. The correction was not applied to the cone counts, for the apparent multiplication referred to above was not present with these elements. This was because fragments of cones did not stain densely nor did they show the granulated appearance which makes fragments of nuclei very clear.

\section{Results}

The untreated counts from the retinae are shown in Figs. 1-3. No correction for the $23 \%$ linear shrinkage has been applied in these figures. The numbers of cones in $600 \mathrm{sq} . \mu$ counting areas are plotted against eccentricity in Fig. 1, for both Macaca and Saimiri. Each point represents the mean over three eyes of equal numbers of counts in both nasal and temporal directions. The two counters (AC and ER) counted in different sections of each eye, counting at least one section from each eye. Each point is placed on the abscissa at the centre of the strip over which the count was made. Thus the point at $1.0 \mathrm{~mm}$ represents the count taken between 0.95 and $1.05 \mathrm{~mm}$ from the fovea.

The numbers of ganglion cells counted in areas of 600 sq. $\mu$ are shown in Fig. 2. Each point represents the average of counts taken in both directions from the five eyes by both counters, for the species indicated. The counts shown in this figure are as obtained, before correction by the Abercrombie fraction of 0.545 . Figures

* For comparison, it should be mentioned that Vakkur (cited in Stone, 1965, as a personal communication) estimated the p.n.d. in one eye of Macaca as $11.0 \mathrm{~mm}$. 


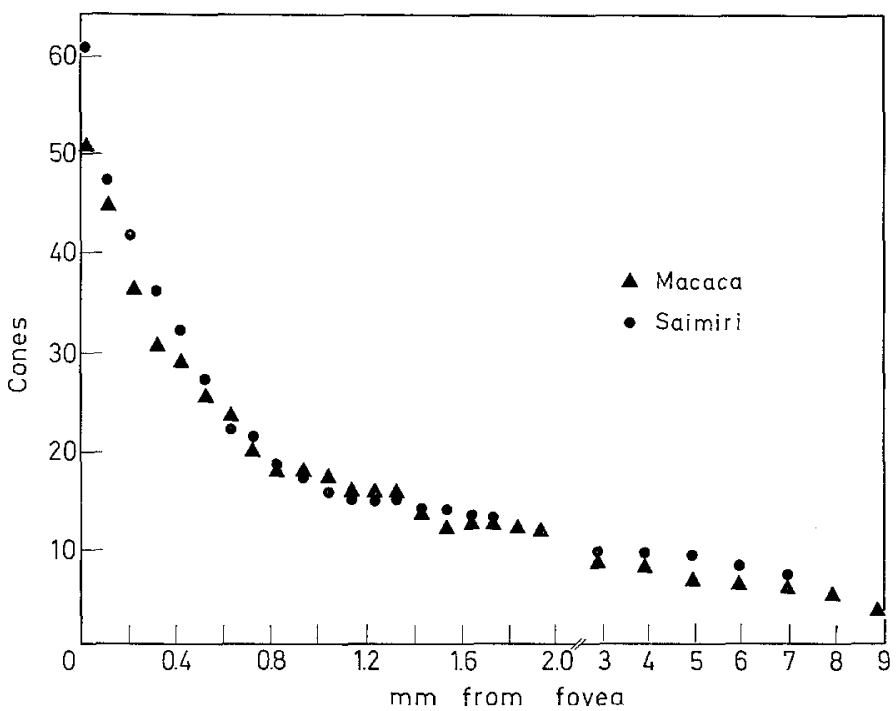

Fig. 1. Cone counts. Ordinate: average number of cones in $600 \mathrm{sq} . \mu$ along nasal and temporal meridia. Abscissa: distance from centre of fovea in $\mathrm{mm}$

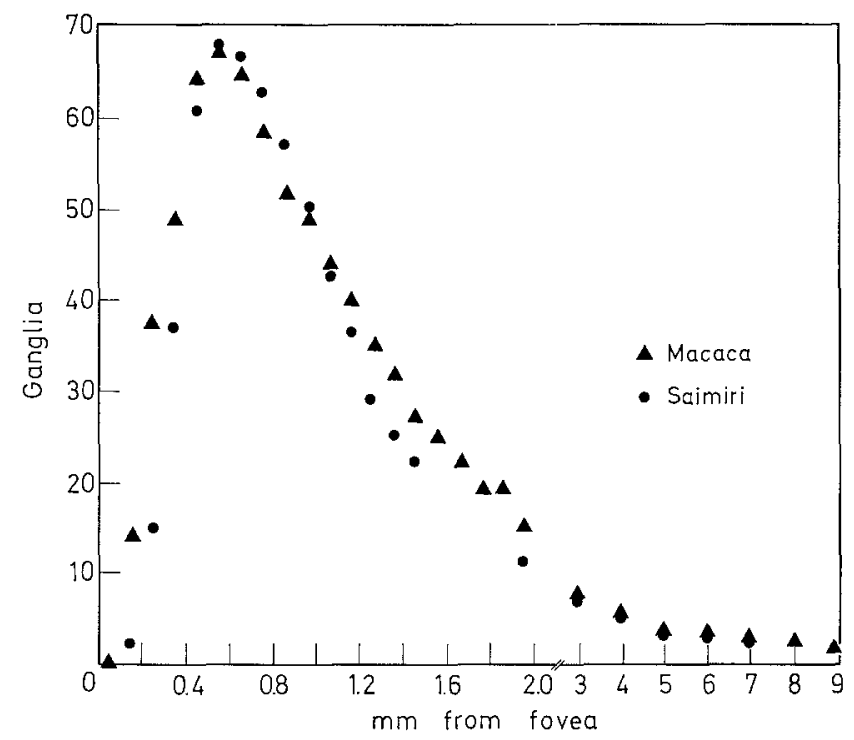

Fig. 2. Ganglion cell counts. Ordinate: average number of nuclei in 600 sq. $\mu$ along nasal and temporal meridia. Abscissa: distance from centre of fovea in $\mathrm{mm}$

3a and $b$ show ganglion cell counts separated with respect to meridian - either nasal or temporal.

The outstanding feature of these raw counts is the similarity in the counts obtained from the two species. The main differences are that cone density is greater in Saimiri than in Macaca between 0.0 and $0.5 \mathrm{~mm}$ and beyond $3.0 \mathrm{~mm}$; and that the foveal excavation of ganglion cells is wider in Saimiri; also ganglion cell density is smaller between 1.0 and $2.0 \mathrm{~mm}$ in Saimiri than in Macaca. 


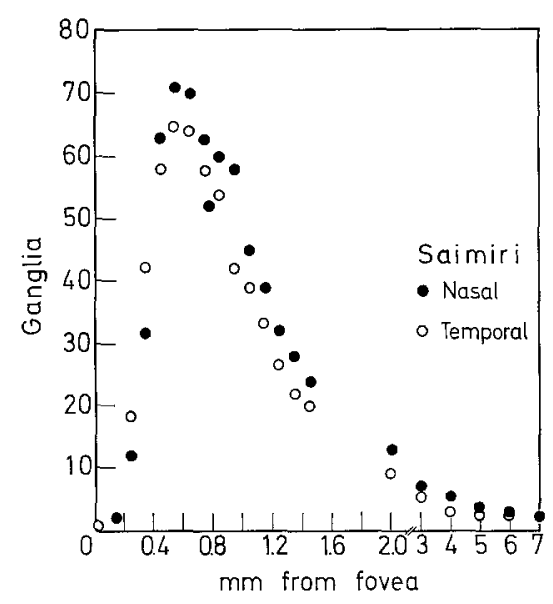

Fig. $3 \mathrm{a}$

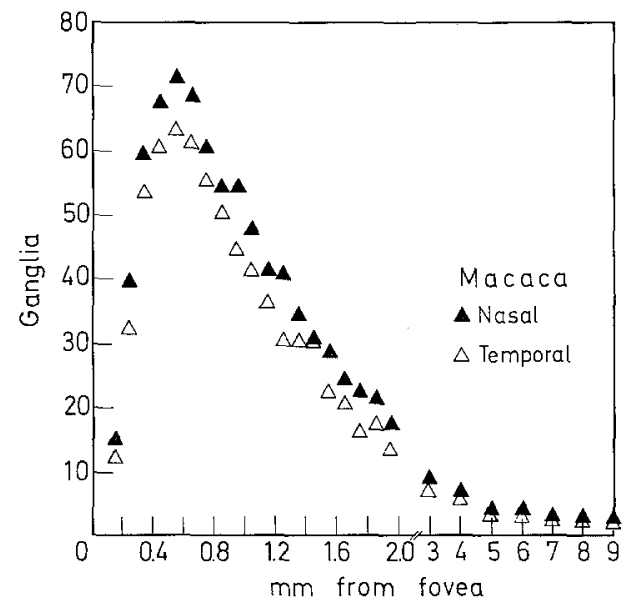

Fig. $3 \mathrm{~b}$

Fig. 3. Ganglion cell counts. As Fig. 2, except that open circles indicate temporal, and closed circles nasal, counts. a. Saimiri, b. Macaca

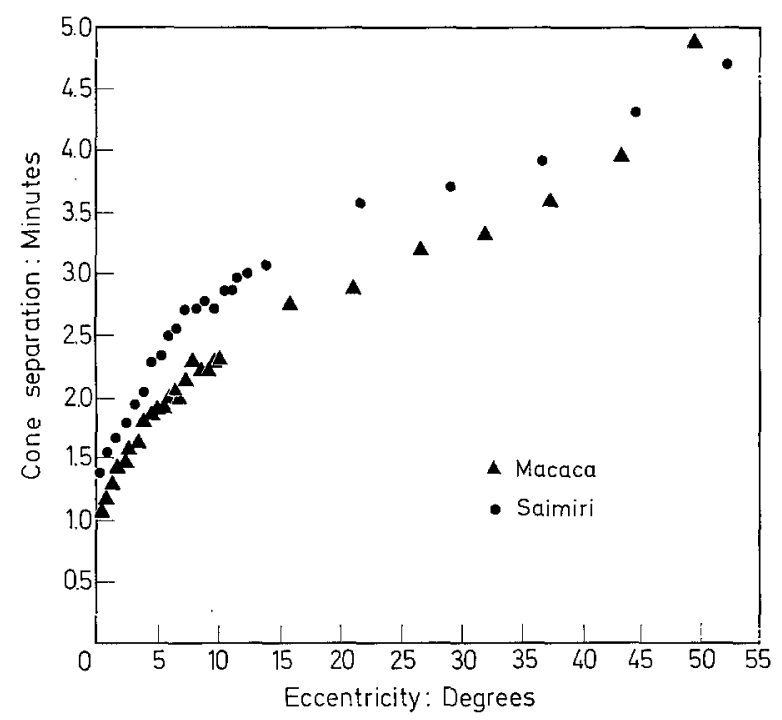

Fig. 4. Cone separation (see text) as a function of eccentricity from the fovea

Cone and g.c. separations, calculated as described in the method, are plotted as functions of eccentricity in degrees in Figs. 4 and 5, which include all points shown in Figs. 1 and 2.

It is clear that Saimiri, with a smaller eye but an acuity similar to that of Macaca, has a greater number of cones per unit area in the fovea (i.e. from 0.0 to $0.1 \mathrm{~mm}$ ). Further counts for the three eyes across the fovea (from $0.05 \mathrm{~mm}$ nasal to $0.05 \mathrm{~mm}$ temporal) gave mean foveal cone densities of 62.8 and 51.5 cones $/ 600 \mu^{2}$ for Saimiri and Macaca respectively. In angular measures, the counts represent cone separations of 1.33 and $1.08 \mathrm{~min}$, for the squirrel monkey and rhesus monkey. These angular figures are in the same direction as the acuity thresholds $-0.74^{\prime}$ 


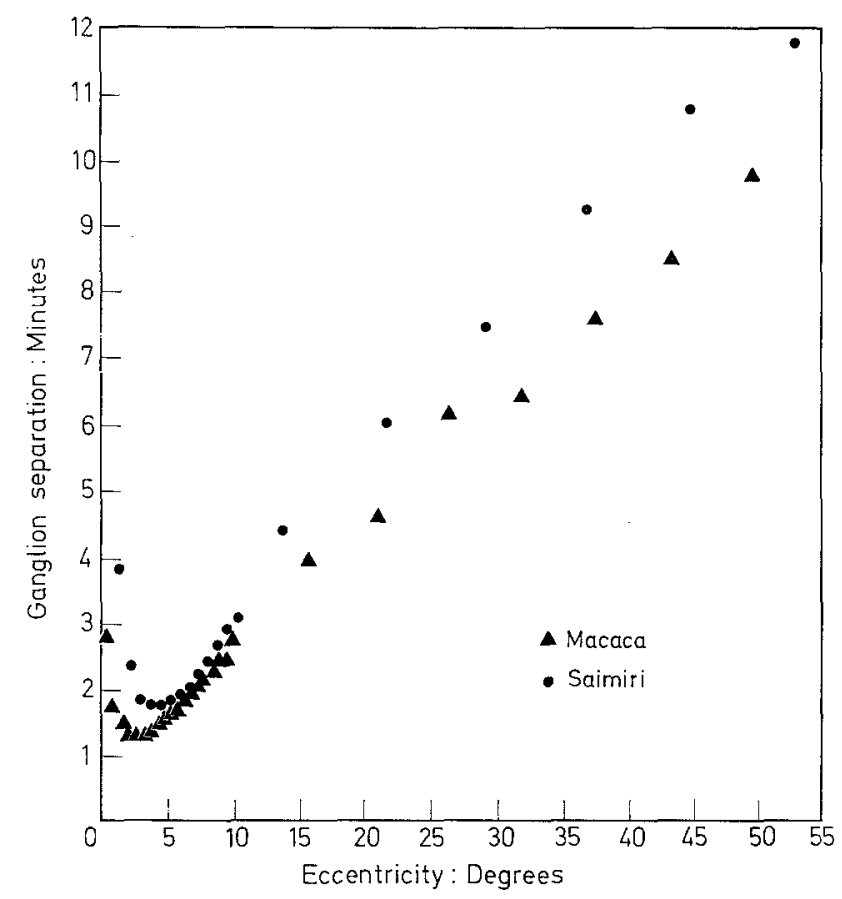

Fig. 5. Ganglion cell separation (see text) as a function of eccentricity from the fovea

for the squirrel monkey and $0.65^{\prime}$ for the rhesus monkey (Cowey and Ellis, 1967). Expressed as ratios the visual acuity of Macaca and Saimiri is $1: 1.14$; the foveal cone separation is $1: 1.23$.

It is more difficult to compare the results for central ganglion cells, because of the foreal excavation of the cells. The width of the foveal excavation is greater, and the ganglion cell density at $3-4^{\circ}$ is smaller in Saimiri than in Macaca (see Fig. 5). Fibres from the bipolar cell layer certainly sweep eccentrically, and g.c.s. situated within $1-4^{\circ}$ eccentricity and possibly further, must be involved in the transmission of information from the fovea.

Cone separation is greater peripherally in Saimiri than in Macaca. Similarly, a difference in peripheral ganglion cell separation was found, with Saimiri showing a greater separation between $5^{\circ}$ and $20^{\circ}$ eccentricity. These differences correlate with the poorer peripheral acuity of Saimiri.

The Projection of the Visual Field on to the Striate Cortex in Saimiri and Macaca

The projection of the visual field on to the striate cortex of Saimiri has been mapped using the short latency $(35-60 \mathrm{msec})$ potential evoked at the cortex by a flash of light (Cowey, 1964). Using the results shown in Fig. 4 of Cowey (1964) the reciprocal of the cortical magnification factor, that is, the number of degrees of visual field mapped linearly on to $1 \mathrm{~mm}$ of cortex, has been calculated for different eccentricities. The results are shown in Fig. 6. No radial asymmetry was found. The line through the points was drawn by eye, and this line is replotted in Fig. 7 . To check the accuracy of the results, the area of the striate cortex of one hemisphere was calculated from the points shown in Fig. 7 by the method described by 


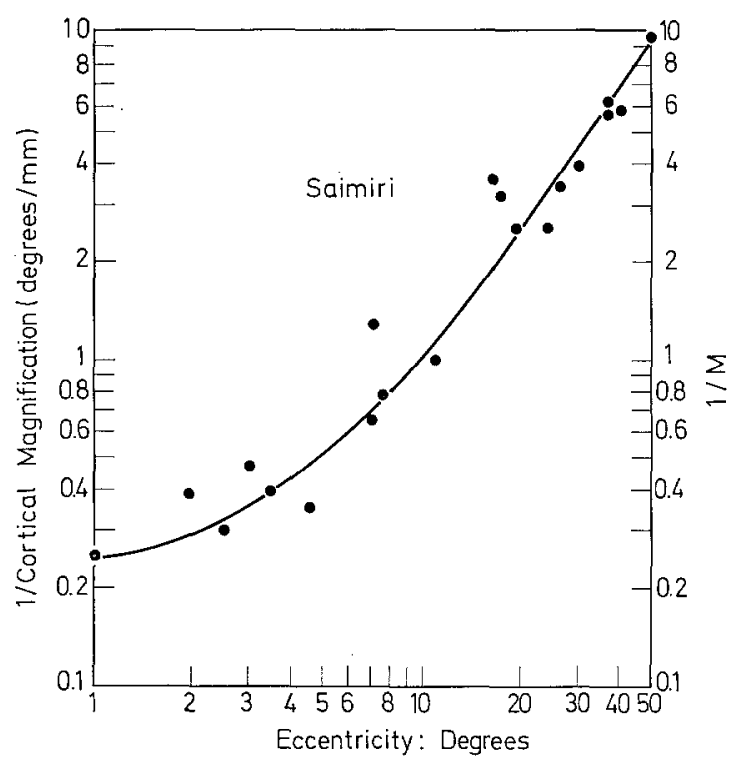

Fig. 6. Reciprocal of the cortical magnification of the visual feld for Saimiri as a function of eccentricity. The line was drawn by eye

Daniel and Whitteridge (1961), which assumes radial symmetry. The areas between selected near semicircles of latitude are determined by first calculating the lengths of the two semicircles on the cortex. The length of any given semicircle is $180 \mathrm{M} \sin \Theta$, where $\Theta=$ eccentricity in degrees and $\mathrm{M}$ is the magnification factor in $\mathrm{mm} / \mathrm{o}$ at $\Theta$. The average of the lengths of the two semicircles is multiplied by the distance along any one meridian between the semicircles: this distance is $\frac{\left(M_{1}+M_{2}\right)}{2}\left(\Theta_{1}-\Theta_{2}\right)$, where $M_{1}$ and $M_{2}$ are the magnification factors at $\Theta_{1}$ and $\Theta_{2}$ degrees. The total area of cortex is the sum of the areas between the different semicircles of latitude. The result was $800 \mathrm{~mm}^{2}$, which agrees well with the average value of $722 \mathrm{~mm}^{2}$ found by direct measurement of the striate area by Cowey (1964). This is an indication that the points for Saimiri shown in Fig. 7 are accurate.

The striate projection of the visual field in a group of monkeys consisting of rhesus, baboons, one cynomolgus and one vervet has been described by Daniel and Whitteridge (1961), who presented their results both as the magnification factor, and as its reciprocal, at different eccentricities. A line has been drawn through their original results in the same manner as in Fig. 6 for Saimiri, and the line replotted at the same eccentricities as for Saimiri in Fig. 7. As a check on this procedure the striate area of the rhesus and other monkeys was calculated using this line and the same eccentricities as for Saimiri. The calculated area was $1530 \mathrm{~mm}^{2}$, which agrees reasonably with the area of $1400 \mathrm{~mm}^{2}$ measured directly (Cowey, 1964).

A comparison of the two curves for the reciprocal of $M$ at different eccentricities shows that between $1^{\circ}$ and $20^{\circ} \mathrm{l} / \mathrm{M}$ (that is, the number of degrees of visual field per $\mathrm{mm}$ of cortex) is greater for Saimiri than for $M a c a c a$, and that $1 / \mathrm{M}$ increases more rapidly in this region for Saimiri. This may be correlated with the slightly poorer foveal acuity of Saimiri, and the greater decline in its acuity as the eccen- 


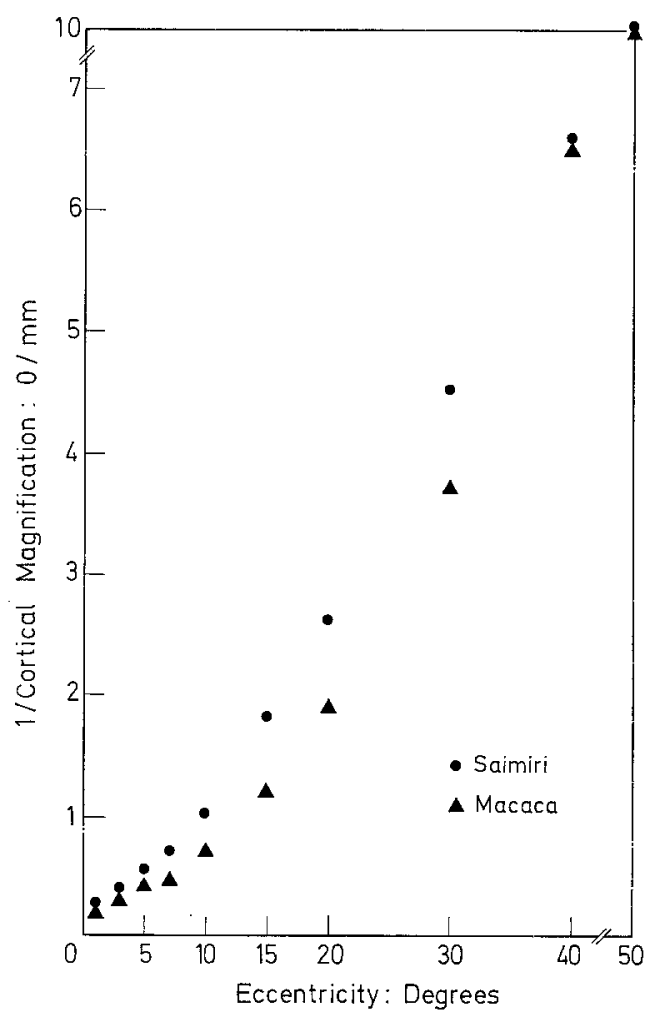

Fig. 7. Averaged reciprocal of $M$ as a function of eccentricity for Saimiri and Macaca

tricity is increased to $2^{\circ}-3^{\circ}$. Values of $1 / \mathrm{M}$ become inseparable for the two species beyond $40^{\circ}$.

\section{The Relationship Between Ganglion Cell Density and Cortical Magnification}

Both the cortical magnification and the ganglion cell density outside the fovea decrease monotonically with increasing eccentricity. Thus retinal topography may in some way match the final magnification given to the visual field. Polyak (1932) on the basis of degeneration experiments referred to by Daniel and Whitteridge (1961), suggested that a mathematical projection of the retina on to the cerebral cortex exists.The results given here allow this suggestion to be tested in Macaca and Saimiri.

The cortical magnification at different eccentricities is plotted as a function of ganglion cell density (number of ganglia in one solid degree) in Fig. 8 for Saimiri and in Fig. 9 for Macaca. The results indicate that between $50^{\circ}$ and $10^{\circ}$ the cortical magnification is approximately proportional to the ganglion cell density. At eccentricities of less than $10^{\circ}-7^{\circ}$ the ganglion cell density cannot be related accurately to eccentricity because of the foveal excavation of ganglion cells, which produces an artificially low density close to the fovea (between about $0^{\circ}$ and $3^{\circ}$ ), and an artificially high density for a few degrees beyond about $3^{\circ}$. Thus the foveal excavation of ganglion cells accounts at least in part for the U-shaped function which relates cortical magnification to ganglion cell density at eccentricities of less than $10^{\circ}$. 


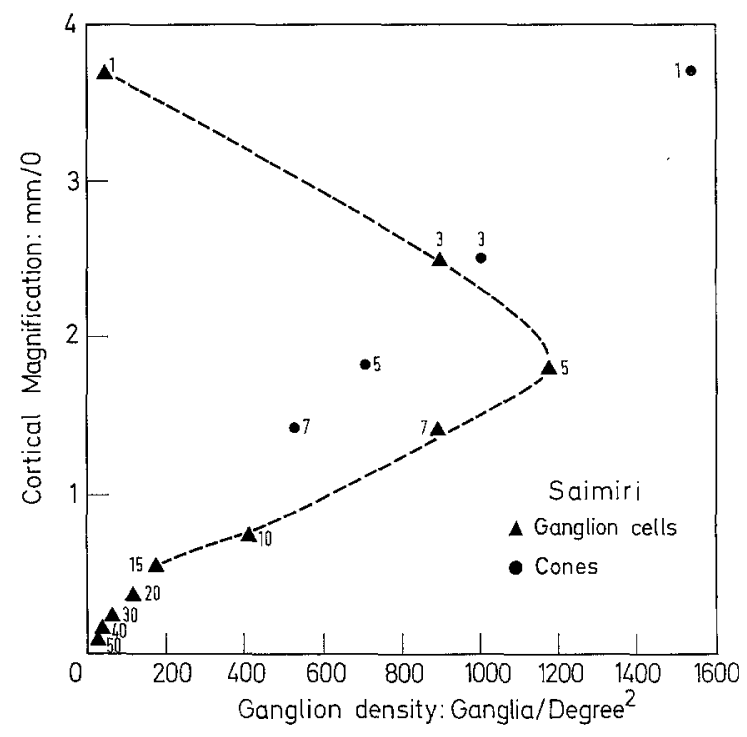

Fig. 8. Cortical magnification as a function of ganglion cell density (number of ganglia per solid degree) for Saimiri. The numbers beside the points refer to the eccentricity in degrees. The dotted line connects ganglion cell points between $15^{\circ}$ and $1^{\circ}$. At eccentricities of $7^{\circ}$ and less, cortical magnification is also plotted as a function of cone density (closed circles, number of cones per solid degree)

An indication of the possible ganglion cell density at low eccentricities was made from the cone density, on the assumption that near the fovea the ganglion cell/cone ratio is about one. In Figs. 8 and 9 the cone density found in this study has been used as an estimate of ganglion cell density at low eccentricities, and is plotted at eccentricities of $7^{\circ}$ and less. It is clear that the cone points plotted continue the approximately linear relation between cortical magnification and ganglion cell density. Thus if the estimates of cone density are accurate relative to the ganglion cell densities and if the ratio of ganglion cells to cones is one near the fovea, the graphs show that cortical magnification is approximately proportional to ganglion cell density between $50^{\circ}$ and $1^{\circ}$ eccentrically. The constant of proportionality is similar in the two species, although of course points for the same eccentricities in the two species come at different places along the line of proportionality. The relation between cortical magnification and cone density is not continued at eccentricities greater than $10^{\circ}$ : if plotted, the points incline sharply towards the 'density' axis - cone density at $40^{\circ}$ is still $220 /$ degree $^{2}$ in Saimiri.

If there were insufficient ganglia at for example $7^{\circ}$ to allow a ratio of one ganglion cell to one cone, then the relationship found above between ganglion cell density and cortical magnification would be in doubt at low eccentricities. To examine this, the cumulated numbers of cones and ganglion cells at different eccentricities is considered in the next section.

Cumulated Numbers of Cones and Retinal Ganglion Cells at Different Eccentricities

Using the average cone and ganglion cell densities between eccentricities, for which points are shown in Figs. 8 and 9, and the retinal solid angle between these 


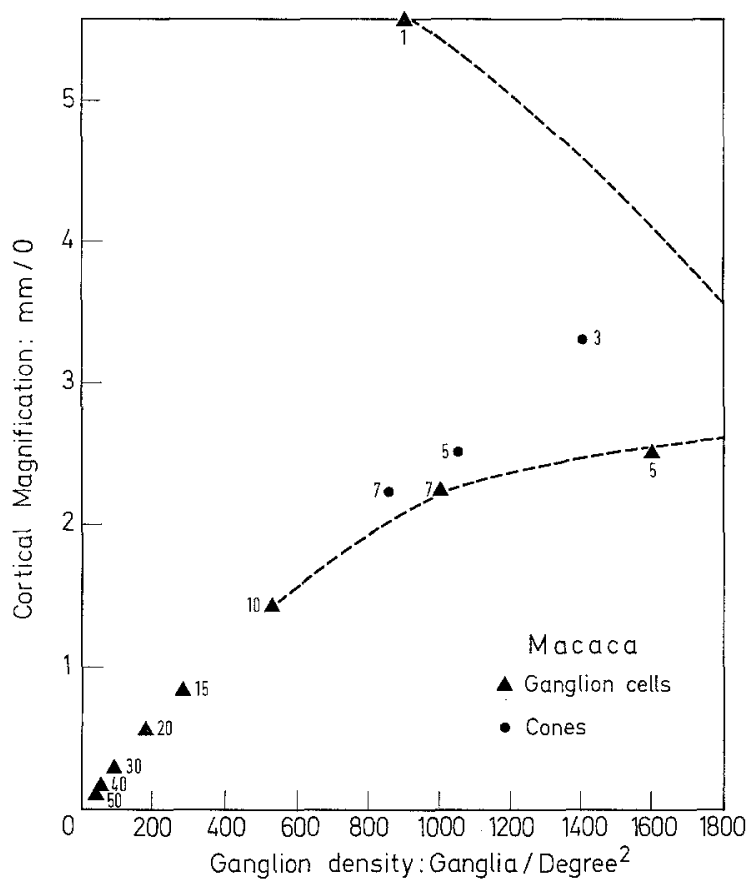

Fig. 9. Cortical magnification as a function of ganglion cell density for Macaca. Legend and conventions as Fig. 8. The ganglion cell density at $3^{\circ}$ is 2300 ganglia per solid degree

eccentricities, the numbers of cones and retinal ganglion cells cumulated at the eccentricities are shown in Figs. 10 and 11. It is clear that there are more ganglion cells than cones between $0^{\circ}$ and $10^{\circ}$ in both species.

Therefore, there are sufficient ganglion cells to make the estimate of ganglion cell density from cone density at eccentricities up to $7^{\circ}-10^{\circ}$ seem reasonable. Two remaining possibilities, which cannot be rejected at present, are that there is a high ratio of ganglion cells to cones very close to the fovea, making a ratio of 1 ganglion cell to 1 cone at $7^{\circ}$ impossible; and that there are insufficient ganglion cells at $7^{\circ}$ for a $1: 1$ ratio because many ganglion cells at this and lower eccentricities are concerned exclusively with rods.

It should be made clear that the calculation of the number of cones and ganglion cells within certain eccentricity limits necessary for Figs. 10 and 11 assumes that the average ganglion cell or cone density within the eccentricity limits is the average of the densities along the nasal and temporal meridia. This method allows a comparison of cones and ganglion cells within and across species, but the absolute figure for cumulated numbers is high because the distribution is not completely radially symmetrical (Whitteridge, 1965): the density along the superior and inferior meridia is less than the average along the nasal and temporal meridia.

\section{Discussion}

Cutting $6 \mu$ horizontal sections of the retinae was chosen in preference to viewing flattened retinae tangentially because of the difficulty which occurs with flattened preparations in counting the stacked ganglion cells near the fovea. By 


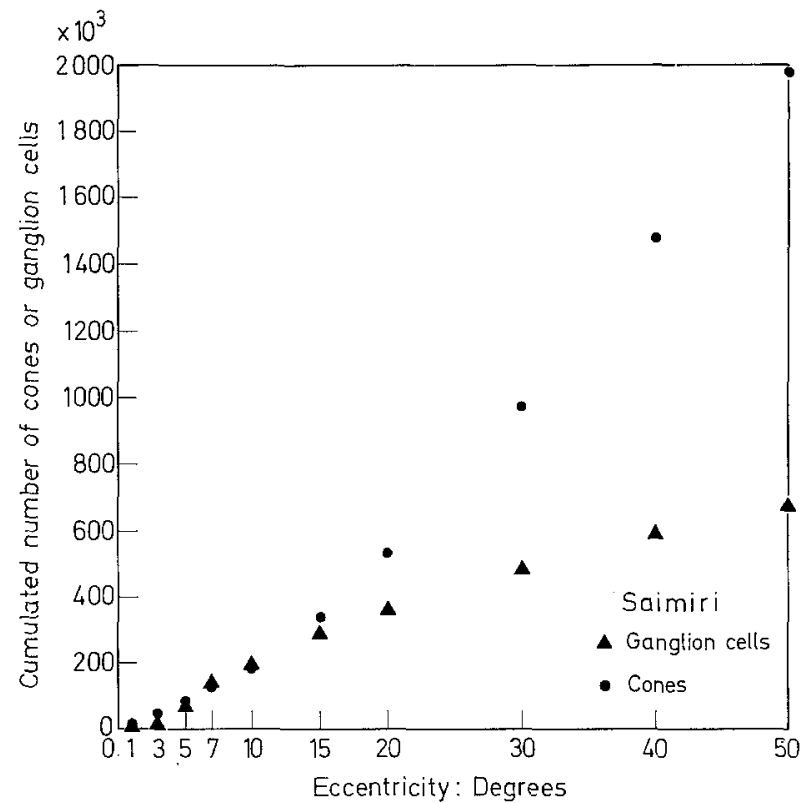

Fig. 10. Cumulated numbers of cones and retinal ganglion cells as a function of eccentricity from the fovea in Saimiri

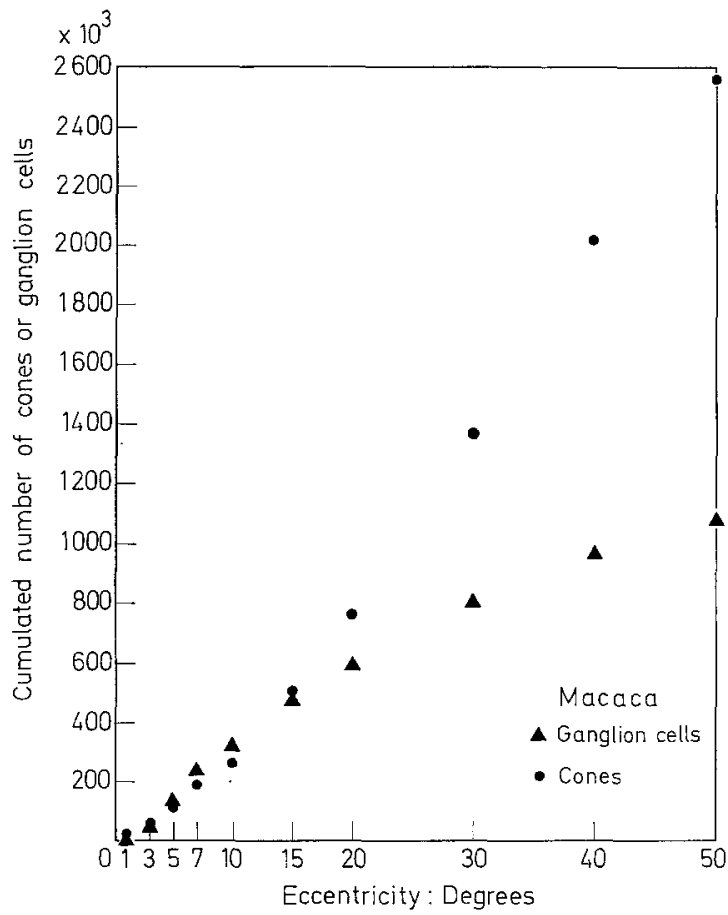

Fig. 11. Cumulated numbers of cones and retinal ganglion cells as a function of eccentricity from the fovea in Macaca 
cutting tangential sections of flattened human retinae Van Buren (1963) estimated ganglion cell density near the fovea by multiplying the count in one $15 \mu$ section by the number of layers of ganglion cells in the region of the count. This would lead to inaccuracies of ganglion cell density in monkeys, because clear layering of ganglion cells is not present. We think it would be difficult to count ganglion cells in one layer only, even if separable layers were present.

The method we chose was not free from difficulties. Ganglion cell nuclei were counted in $6 \mu$ sections, and as the average diameter of the nuclei is little less than this, Abercrombie's correction (Abercrombie, 1946) was used. For reasons stated under Methods, Abercrombie's correction was not applied to the cone counts. If this is incorrect cone density will be less than that given throughout the results.

The estimates of average cone separation plotted in Fig. 4, obtained by taking the reciprocal of the square root of angular density, could be made more absolutely rather than relatively accurate by consideration of the 'packing fraction'. This has not been done since the cone separations are accurate relative both to each other and to ganglion cell separations, which were calculated by the same methods.

When counting cell nuclei in the ganglion cell layer of the retina, it was difficult to distinguish ganglion cells from neuroglial cells. All spherical nuclei with a dia. meter of $5-9 \mu$ in layer 8 were counted and included in the ganglion cell count. Occasional smaller, rather irregular nuclei with a diameter of $1.5-3 \mu$ were seen, and these nuclei stained very darkly. It was assumed that these nuclei belonged to neuroglial cells and they were ignored. But it is possible that some neuroglial cell nuclei were indistinguishable from ganglion cell nuclei, and were included in the counts. The separation of these two cell types presented a difficulty for Ramón y Cajal (Polyak, 1941, p. 356).

Figure 3 shows that the ganglion cell count along the nasal meridian is consistently greater than along the temporal meridian in both Macaca and Saimiri. Van Buren (1963) noted the same asymmetry in both rhesus monkeys and man. The ganglion cell distribution is similarly asymmetrical in the baboon (Whitteridge, 1965). The contour map for the baboon shows also that inferior and superior ganglion cell distributions are more like the temporal than the nasal distribution. This may mean that in Figs. 10 and 11 the cumulated number of ganglion cells is a little high because the calculation assumed that the ganglion cell density at a given eccentricity over the whole eye is the mean of nasal and temporal densities.

A similar type of asymmetry was found in cone counts. In both Macaca and Saimiri, the cone count is about $14 \%$ greater nasally than temporally between 0.0 and $0.5 \mathrm{~mm}$. In Saimiri the cone count is about $17 \%$ greater nasally between 3 and $7 \mathrm{~mm}$. The corresponding difference in Macaca is about $23 \%$. Østerberg (1935) found that in man nasal cone density is consistently greater than temporal.

The slightly poorer foveal acuity of Saimiri, compared with Macaca, is associated with a smaller eye diameter and a smaller brain weight (means 26.9 and $78.1 \mathrm{~g}$ respectively, Cowey, 1964). It has been shown here that Saimiri partly compensates for its smaller eye diameter with a greater cone density (cones $/ \mathrm{mm}^{2}$ ) in the fovea. When converted to angular terms, which allows a comparison with the minimal angle of resolution (M.A.R.), the cone separation in Saimiri is greater than that in Macaca, and this correlates with Saimiri's slightly greater M.A.R. at the fovea. The lower cortical magnification of the foveal part of the visual field 
(measured at $1^{\circ}$ ), and the probably greater foveal ganglion cell separation at the fovea, correlate with the greater foveal M.A.R. of Saimiri. The foveal ganglion cell separation has not been estimated conclusively here because of the foveal excavation of ganglion cells.

The greater M.A.R. of Saimiri outside the fovea (at $2^{\circ}-3^{\circ}$ eccentrically) has been shown to be correlated with a greater cone and ganglion cell separation, and a lower cortical magnification, when Saimiri is compared with Macaca.

An investigation of the relationship between the quantitative measures of topography we used shows that, at least peripherally, the cortical magnification of the visual field is proportional to ganglion cell density in Saimiri and Macaca. This indicates that there may be a precise topographical arrangement within the visual pathways. A functional correlate of this topographical arrangement may be visual acuity, for Daniel and Whitteridge (1961) have found that the diminution in the magnification factor from foveal to peripheral projection area in the rhesus monkey is remarkably similar to the fall in visual acuity in man from fovea to periphery. Our qualitative comparisons of acuity and $\mathrm{M}$ in different species of monkey are in line with the suggestion that there may be a close relationship between acuity and cortical magnification. We hope to extend the study using the variation of acuity with eccentricity to determine the way in which the M.A.R. may match the quantitative anatomical patterns found here.

\section{References}

Abercrombie, M.: Estimation of nuclear population from microtome sections. Anat. Rec. 94, $239-247$ (1946).

Cowey, A.: Projection of the retina onto striate and prestriate cortex in the squirrel monkey, Saimiri sciureus. J. Neurophysiol. 27, 366-393 (1964).

- Perimetric study of field defects in monkeys after retinal and cortical ablations. Quart. J. exp. Psychol. 19, 232-245 (1967).

- Ellis, C.M.: Visual acuity of rhesus and squirrel monkeys. J. comp. physiol. Psychol. 64, 80-84 (1967).

- - The cortical representation of the retina in squirrel and rhesus monkeys and its relation to visual acuity. J. exp. Neurol. 24, 374-385 (1963).

Daniel, P.M., Whitteridge, D.: The representation of the visual field on the cerebral cortex in monkeys. J. Physiol. (Lond.) 159, 203-221 (1961).

Duke-Elder, W.S. : Text-book of Ophthalmology, Vol. I. London: Henry Kimpton 1932.

Osterberg, G.: Topography of the layer of rods and cones in the human retina. Acta ophthal. (Kbh.) 65, Suppl., 1-102 (1935).

Polyak, S.: The main afferent fiber systems of the cerebral cortex in primates. University of California Publication in Anatomy, 2. Berkeley: University of California Press 1932.

- The vertebrate visual system. Ed. by H. Klüver. Chicago/Ill.: University of Chicago Press 1957.

Stone, J.: A quantitative analysis of the distribution of ganglion cells in the cat's retina. J. comp. Neurol. 124, 337-352 (1965).

Van Buren, K.M.: The retinal ganglion cell layer. Springfield/Ill.: Charles Thomas 1963.

Weiskrantz, L., Cowey, A.: Comparison of the effects of striate cortex and retinal lesions on visual acuity in monkeys. Science 155, $104-106$ (1967).

Weymouth, F.W.: Visual sensory units and the minimal angle of resolution. Amer. J. Ophthal. 46, 102-114 (1958).

Whitteridge, D.: Geometrical relations between the retina and the visual cortex. In: Mathematics and computer science in biology and medicine. London: Medical Research Council 1965.

Dr. A. Cowey, Institute of Experimental Psychology 1 South Parks Road, Oxford (England) 